\title{
Optimization design of planar transformer in DC-DC converter
}

\author{
Linlin Tana, Xu Yang and Dian Yuwen \\ Xi'an Jiaotong university, School of Electrical Engineering, 710049 Xi'an, China
}

\begin{abstract}
Planar transformer show great advantages compared with traditional transformer in DC-DC converter for its small size and low power dissipation. However, its performance might not be as effective as expected in high frequency and high current applications for the existence of high frequency effects. In this work, the high frequency effects are analyzed and detail optimization design methods for planar transformer winding with interleaved parallel winding arrangement method are introduced, which can help equal the current flowing in the winding and decrease copper loss. Finite Element Analysis (FEA) results are given to verify the design.

Keywords: planar transformer; high frequency effects; DC-DC converter.
\end{abstract}

\section{Introduction}

High operating frequency and low power dissipation are the tendencies and requirements in modern switching power technology, which give great challenge to the design of magnetic components. As a result, the magnetic component design has been a critical issue in the modern industrial area. Conventional transformer has been not suitable for modern switching power supply for its large scale, high losses etc. Compared with traditional transformer, planar transformer has been widely used in the low-voltage and high-current applications for its small value, low loss and excellent thermal characteristic.

However, planar transformer technology meets several issues in high frequency application. High frequency effects are discussed and modelled in [10]. Planar transformer commonly use a PCB or copper foil as winding which can significantly decrease the skin effect in high frequency. But the thin winding also causes larger conduction losses for the increase of copper resistance, especially in large current application. Parallel winding method is usually employed to decrease the copper loss [2, 5-7]. But the current distribution is usually complicated and unequal because of high working frequency and the proximity effect, resulting in high AC resistance [2]. Interleaved parallel winding arrangement proved in this work can significantly reduce this phenomenon and decrease the copper loss as a result.

\section{High frequency effects}

This part will give a brief introduction to high frequency effects that will cause extra power losses in winding conductors of transformer. The specific high frequency effects contain skin effect and proximity effect, which have significant influence on ac resistance of planar transformer windings.

a Corresponding author : sdlytll@163.com 


\subsection{Skin effect}

Figure 1 illustrate a common solitary conductor in which AC current $\mathrm{i}(\mathrm{t})$ flowing through it. According to the Fridays' Law, we can easily get the flux line around the conductor as shown. With changing AC current, the flux will correspond changing in the same law. Lenz's law state that the voltage induces by the changing flux is of the polarity that tends to drive a current through the loop to counteract the flux change [9]. The current $i^{\prime}(t)$ induces by the voltage will then alter the distribution of current in conductors as reduce the net current density in the center of the conductor, and increase the net current density near the surface of the conductor.

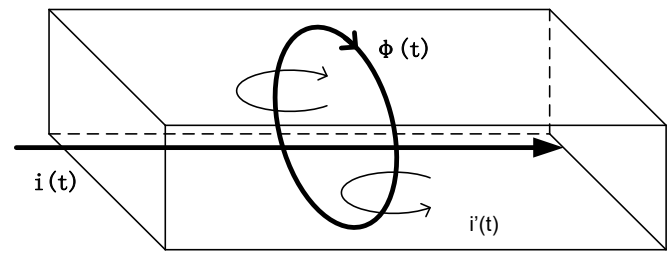

Figure 1. Skin effect model

\subsection{Proximity effect}

Proximity effect presents the phenomena of uneven current distribution within the adjacent conductors. Two conductors that are in close proximity to each other are illustrated in Figure 2, assume that the two conductors are closely enough to ignore the skin effect, in which the conductor (a) carries high frequency ac current $\mathrm{i}(\mathrm{t})$ and conductor $(\mathrm{b})$ carries no current.

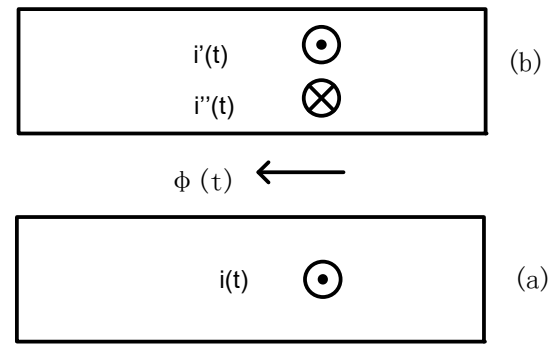

Figure 2. Proximity effect model

However, the actual current flowing in conductor (b) will not be zero because of the eddy current induced by $i(t)$. As discussed above, the flux induced by $i(t)$ will existed between the conductor (a) and (b), which will generate eddy current in the bottom side of conductor (b) according to Lenz's Law. We have known that the current flowing through conductor (b) is zero, as a result, a corresponding current $i$ '' $(t)$ that will be equal and opposite to $i$ ' $(t)$ will be induced. For transformer winding, the situation may be worse for the existence of multi-lays.

All the high frequency effects will induce the uneven distribution of current flowing through the transformer winding and increase the resistance and copper loss of transformer winding. The optimization design will focus on those effects that are inevitable.

\section{Optimization design and Finite Element Analysis}

To verify the effectiveness of optimization design of planar transformer, parallel and interleaved parallel winding arrangement methods are comparative analyzed in this part. Figure 3, 4 modelled four planar transformers by Finite Element Analysis, in which the winding arrangement is given and the core of transformer is concealed for better clarification. All the winding models have PCB layers 
with $0.1 \mathrm{~mm}$ thickness and $0.1 \mathrm{~mm}$ insulation spacing. The current excitation is added to corresponding winding.

\section{1 parallel winding of $\mathrm{PCB} / \mathrm{copper}$ foil layers}

\subsubsection{Parallel winding analysis}

Current handling capacity of one PCB or copper foil layers is limited for the high loss in large current application. Common winding arrangement with only one single-layer will not be suitable for the huge current density and copper loss it may cause. So the parallel connection of PCB or copper foil layers in secondary winding is employed here to enhance the current handling capacity of winding and optimize the performance of planar transformer [3]. With the parallel winding method, the current flowing through the secondary winding will decrease markedly and the copper loss will then be reduced as a result.

\subsubsection{Finite Element Analysis (FEA) results}

The Finite Element Analysis results are given here to verify the methods. Ansys Maxwell 2D Field Simulator which has been used widely to analyze the electromagnetic field is employed here to give the simulation results. Figure. 3 shows the simulation results where two different conditions are given to make a contrast between common winding arrangement and the parallel method.

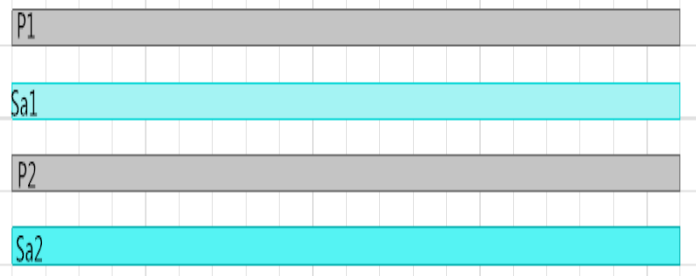

(a)

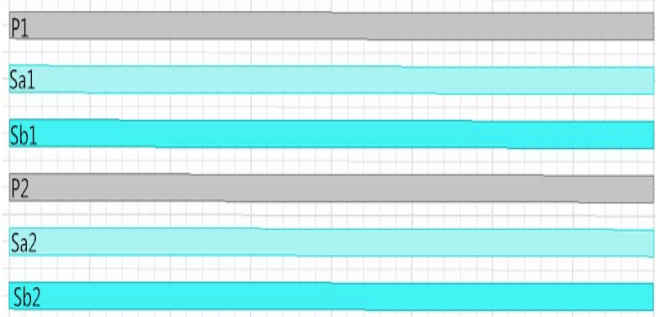

(c)

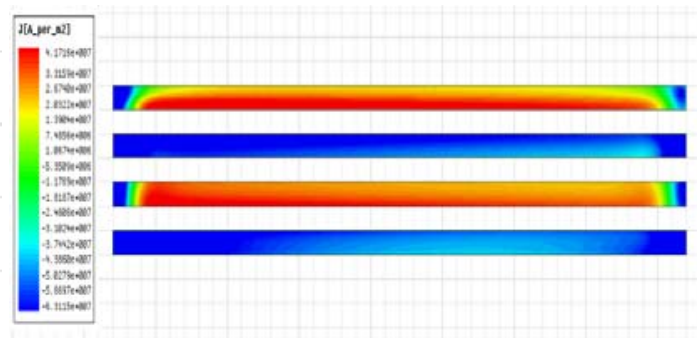

(b)

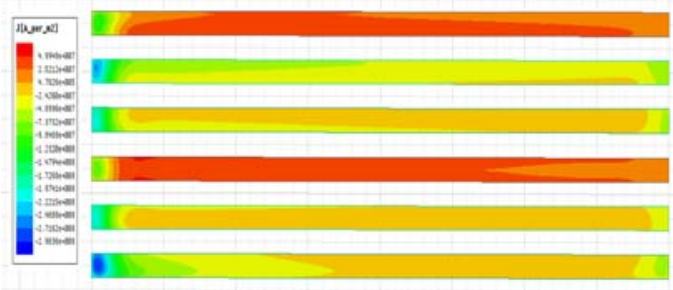

(d)

Figure 3. Parallel winding analysis

Figure 3 gives a case study of 2:1:1 centre-tapped transformer's Finite Element Analysis results to verify the advantages of parallel winding arrangement method. The Figure 3(a) and 3(c) are the winding arrange method for parallel winding or not. P1 and P2 is the primary winding, Sa and Sb is the secondary winding while $\mathrm{Sa} 1, \mathrm{Sa} 2, \mathrm{Sb} 1, \mathrm{Sb} 2$ in figure 3(c) means the parallel connection in secondary winding.

Figure 3(b) and 3(d) are the simulation results of Finite Element Analysis (FEA). As shown in Figure 3(b) and 3(d), the current density in common arrangement can achieve $6 \times 10^{7} \mathrm{~A} / \mathrm{mm}^{2}$ while less than $4 \times 10^{7} \mathrm{~A} / \mathrm{mm}^{2}$ in parallel winding arrangement, which verify the conclusion that parallel arrangement can reduce the winding's current density in planar transformer. 


\subsection{Interleaved winding arrangement for centre-tapped planar transformer}

\subsubsection{Interleaved winding arrangement analysis}

Centre-tapped transformer is widely used in isolation circuits. For centre-tapped transformer, the secondary winding commonly work on time-sharing mode, take the secondary winding 1 work as example, which means that all current will flowing through the secondary winding 1 while the secondary winding 2 will handle no current.

Thus the condition will be complicated as that has been analyzed in part 2. Current flowing in the secondary winding 1 will induce the variation of electromagnetic field between two winding, which will cause the eddy current existed in the secondary winding 2 . As a result, the winding that is not work will also exist eddy loss. Interleaved winding arrangement for centre-taped planar transformer will significantly reduce this impact for the isolation between two time-sharing work winding [6].

\subsubsection{Finite Element Analysis (FEA) results}

Figure 4 shows the Finite Element Analysis (FEA) results where two different conditions are given to make a contrast between the common winding arrangement and interleaved winding arrangement for centre-tapped planar transformer.

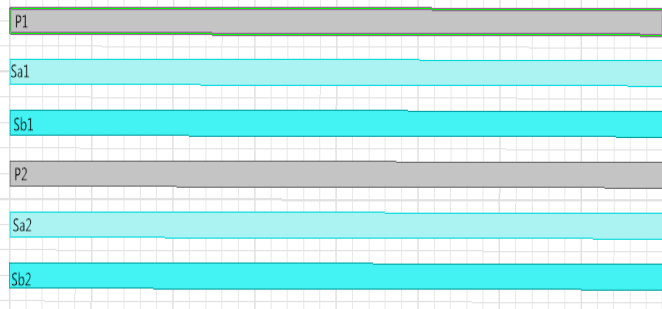

(a)

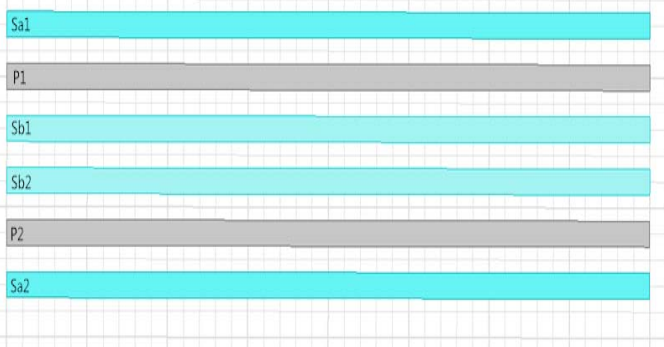

(c)

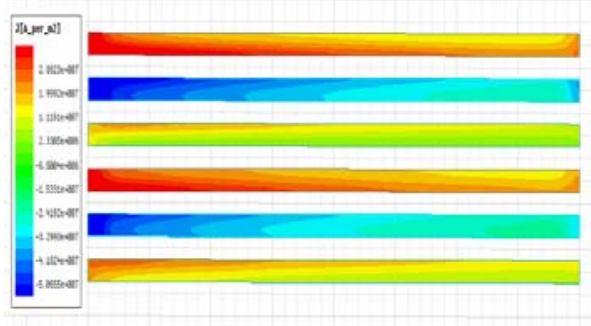

(b)

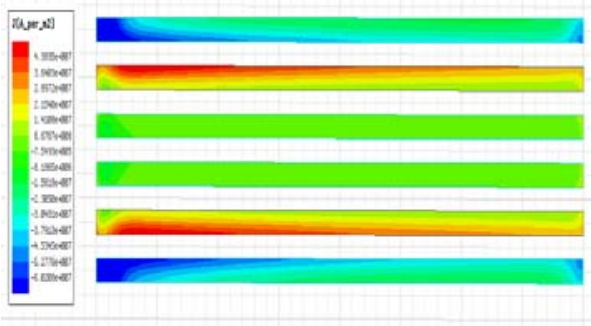

(d)

Figure 4. Interleaved winding arrangement

Figure 4 gives a comparison simulation results between common winding connection and interleaved winding connection method in the case of 2:1:1 centre-trapped transformer. Figure 4 (a) is common winding arrangement and Figure 4 (c) is interleaved winding arrangement for centre-trapped $\mathrm{PCB}$ or cooper foil winding. P1 and P2 is the primary winding, Sa and Sb mean the secondary winding working on time-sharing mode.

Figure 4 (b) shows the simulation results of common winding arrangements. The current excitation is given to winding $\mathrm{P}, \mathrm{Sa} 1$ and $\mathrm{Sa} 2$ while winding $\mathrm{Sb} 1$ and $\mathrm{Sb} 2$ have no excitation. We can get the result that the current density of $\mathrm{Sb} 1$ and $\mathrm{Sb} 2$ is not zero though there is no excitation applied to them, which prove the existence of eddy and proximity effect. Figure 4 (d) shows the result when interleaved winding arrangement is applied on the centre-trapped transformer. The result shows apparently that with interleaved winding arrangement, the current flowing in the winding $\mathrm{Sb} 1$ and $\mathrm{Sb} 2$ 
is nearly zero, which verifies the effectiveness of interleaved winding arrangement for centre-trapped transformer.

\subsection{Maxwell 3D Verification Simulation}

Maxwell 3D simulation mode is built here to verify the whole performance of centre-trapped planar transformer with interleaved parallel winding. Figure 5(a) is the simulation model while Figure 5(b) is the current density distribution results of winding. The core material property is set by B-H curve and the winding are interleaved with 6 series primary winding and 4 secondary winding with 3 lays parallel respectively. The current density distribution among the transformer core is relative uniform, which verify the whole optimization design.

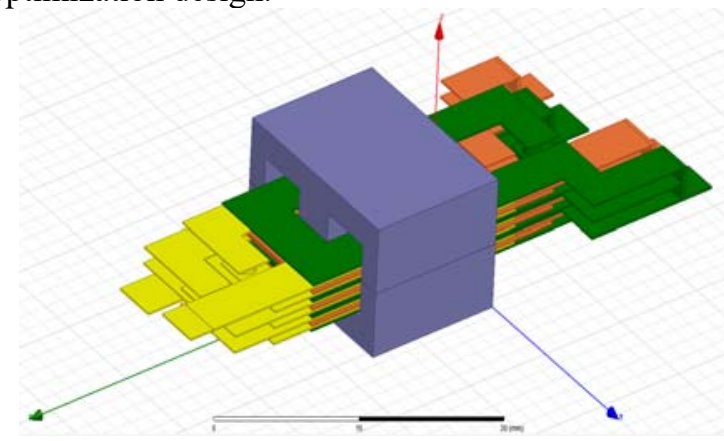

(a)

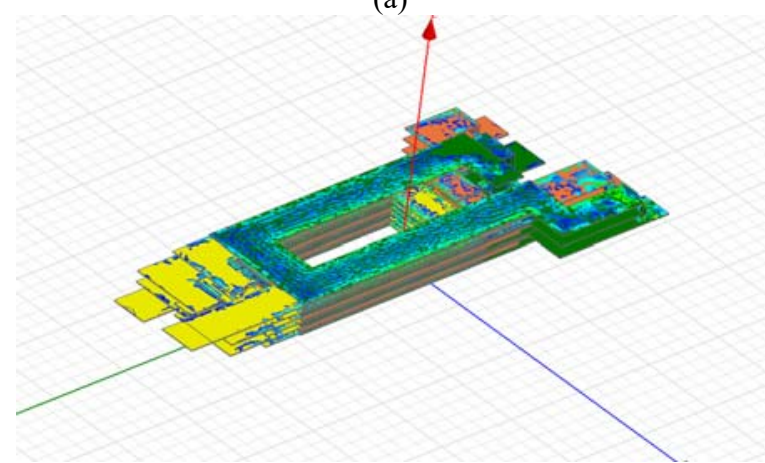

(b)

Figure 5. Maxwell 3D simulation mode

\section{Conclusion}

High frequency and high current have been the main application occasion for planar transformer, which will induce high current density and uneven current distribution [10]. Parallel winding and interleaved winding arrangement method is revealed in this paper to show great advantages for planar transformer, especially for the centre-trapped planar transformer. Specific analysis and contrast is given by Finite Element Analysis (FEA) to show the effectiveness of optimization design compared with common winding arrangement methods. A whole Maxwell 3D model is built finally to verify the optimization design with interleaved parallel centre-trapped planar transformer.

\section{References}

1. Wei Chen, Yipeng Yan, Yuequan Hu and Qing Lu, Model and design of PCB parallel winding for planar transformer, Magnetics, IEEE Transactions, vol. 39, no. 5, pp. 3202-3204, (2003) 
2. P. R. Prieto, J. A. Cobos, O. Garcia, P. Alou, J. Uceda, Using parallel windings in planar magnetic components, Proc. IEEE PESC'01 Conf., pp.2055-2060

3. Z. Ouyang, O. C. Thomsen and M. A. E. Andersen, Optimal design and tradeoff analysis of planar transformer in high-power DC-DC converters, IEEE Trans. Ind. Electron., vol. 59, no. 7, pp. 2800-2810, (2012)

4. G. Wang and H. Yang, Optimal design of high-power, mid-high frequency transformer, in proc. International Conference on Electronic Mechanical Engineering and Information Technology, pp. 2792-2795, (2011).

5. Kuang Jian-jun, Ruan Xinbo, Ren Xiaoyong, Consideration of Current Sharing Among Parallel Windings of Central-tapped Transformer, Proceedings of the CSEE, 29(9):112-117, (2009)

6. Prieto R, Cobos J A, Garcia $\mathrm{O}$, and et al. Using parallel windings in planar magnetic components. in Proc. IEEE PESC, Vancouver, Canada, 2001: 2055 -2060.

7. Hu Y, Guan J, Bai X, and et al. Problems of paralleling windings for planar transformers and solutions. in Proc. IEEE PESC, Cairns, Australia: 597-601, (2002).

8. Pollock J D, Sullivan C R. Modeling foil winding configurations with low AC and DC resistance. in Proc. IEEE PESC, Recife, Brazil: 1507 -1512,(2005).

9. Erickson. Fundamentals of Power Electronics. Fundamentals of power electronics /. 508-530.

10. Mao Xingkui, Chen Wei. AC Loss Modeling and Analysis For Parallel PCB Winding in Highfrequency Power Planar Transformer. Proceedings of the CSEE,2006, 26(22):167-173 